\title{
Influence of amount of reward on maze learning in hooded and albino rats'
}

\author{
Henry A. Cross, 2 Richard J. Rankin and John Wilson \\ OKLAHOMA STATE UNIVERSITY
}

\begin{abstract}
Hooded and albino rats learned a Y maze under highand low-reward conditions. The albinos were superior. Ss receiving high reward made fewer errors than Ss given low reward, confirming recent findings that even with the absolute method quantitative variation in incentives influences learning measures.
\end{abstract}

\section{Problem}

Pubols (1960) concluded that quantitative variation in incentives has no influence on time-independent measures of learning unless Ss first have been made to discriminate among amounts of reward, and he reviewed a number of studies which failed to find differences among groups given incentives of different sizes with the absolute method. Only Schrier (1956) found differences when the learning problem was a complex one.

More recently, hooded rats receiving high reward were observed to make fewer errors in a multiple-Y maze than animals receiving low reward (Cross \& Boyer, 1964; Lawson et al., 1959). Since the studies with negative results all used albino rats, a test for possible strain differences was appropriate, particularly since Denny (see Lawson et al., 1959) has stated that hooded rats respond somewhat more differentially to different amounts of reward than do albinos.

The study reported here compared albino and hooded rats in a factorial arrangement of treatments which incorporated variation in both the quantity and quality of reward.

\section{Method}

Ss were 56 naive male rats, 28 hooded and 28 albino, 80 to 100 days of age at the start of the experiment. ${ }^{3}$ Four units of a commercially made (G. H. Wahmann Co.) Warden-Warner multiple-Y maze were utilized. The maze was located in a small room and was positioned in the same place throughout the experiment with no attempt made to avoid differential cues due to shadows in and around different parts of the maze. Illumination in the room was provided by a shaded 40 -watt bulb suspended 5 feet above the middle of the maze.

Ten days before the start of maze training, Ss were placed on 23-hour food deprivation. They were not handled or adapted to the maze before actual training. Each of the 28 Ss of a given strain was randomly assigned to one of four reward conditions: high regular (i. e., an alfalfa-base pellet), high dextrose, low regular, or low dextrose. All incentives were J. P. Noyes 45-milligram pellets, and four pellets constituted the "high" condition, while one pellet defined the "low" condition.
Each $\mathrm{S}$ was given one trial daily in the maze, and training was continued for 15 days. The correction method was employed, but Ss were removed from the maze if they did not complete it within a 4-minute period.

$\mathrm{Cul}$ entries while in the true direction of the goal, and re-entries resulting from reversals of direction, were scored as errors. All analyses were performed on total errors made during each of three 5-day test blocks.

\section{Results}

Differences between strains and reward amounts are apparent in the error data seen in Fig. 1. A four-factor analysis of variance indicated that all main effects were significant: blocks of trials (F, 60.46; df, 2/96; $p<.001)$; strain (F, 32.54; df, 1/48; p <.001); amount (F, 6.93; df, $1 / 48 ; \mathrm{p}<.02)$; and quality (F, 8.77; df, $1 / 48 ; \mathrm{p}<.01)$. Ss receiving destrose made fewer errors than those receiving the regular alfalfa-base pellet. There were several significant interactions: strain $\mathrm{x}$ quality $(\mathrm{F}, 5.61$; df, $1 / 48 ; p<.05)$; amount $x$ quality (F,5.20; df, 1/48; $\mathrm{p}<.05)$; strain $\mathrm{x}$ blocks (F, 10.91; df, 2/96; $\mathrm{p}<.01$ ); and strain $\mathrm{x}$ amount $\mathrm{x}$ quality $\mathrm{xblocks}$ (F, 4.60; df, 2/96; $\mathrm{p}<.02)$.

The hooded rats receiving low regular reward failed to learn, making more errors during Block 2 than Block 1. This result appears to have produced the significant third-order interaction and to have accentuated other differences. An unweighted-means analysis with these seven aberrant Ss removed revealed the following differences: strain (F, 19.52; df, 1/47; p <.001); blocks ( F,

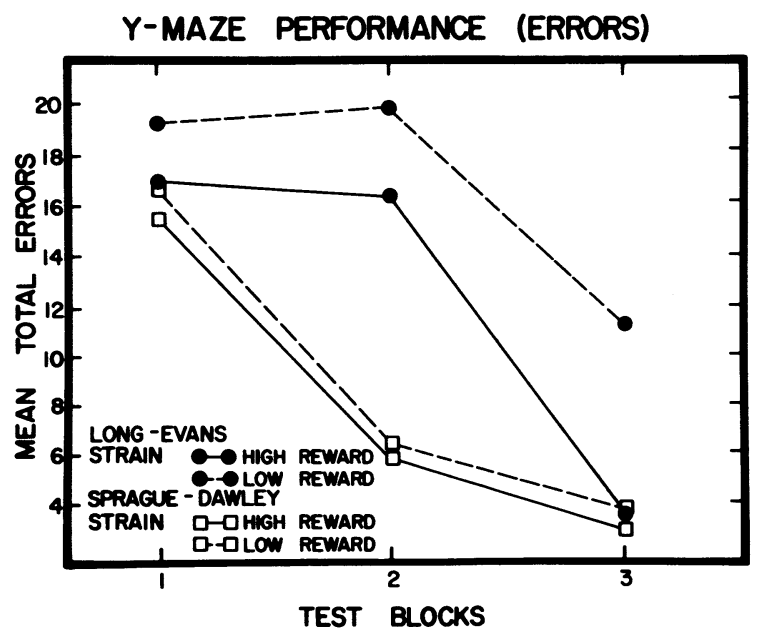

Fig. 1. 
94.95; df, 2/94; p <.001); and strain x blocks (F, 11.03; df, 2/94; $p<.001)$. All hooded Ss displayed extreme emotionality, performing less well than previous well adapted Ss had in the same apparatus (Cross \& Boyer, 1964).

An analysis of the error data for only the albino rats revealed a significant amount effect (F, 5.86; df, 1/24; $\mathrm{p}<.05)$ and a significant blocks effect ( $\mathrm{F}, 121.44$; df, $2 / 48 ; \mathrm{p}<.001)$.

\section{Diseussion}

Although the difficulty encountered with the hooded animals of this study restricts the interpretation which can be made about some variables, the primary question which prompted the investigation has received an unequivocal answer. Albino rats, like hooded animals, show differential learning in a multiple-Y maze as a result of quantitative variation in incentive magnitude. This rules out the possibility that conflicting findings in the area can be attributed to strain differences and redirects the search for the variable (s) of critical importance.

While the generally poor performance of the hooded Ss has accentuated what may be real strain differences in learning, the results are not without practical significance. Experimenters wishing to use hooded Ss in similar experiments should consider the advantages of prior adaptation to the experimental apparatus. When the performance of adapted hooded Ss (Cross \& Boyer, 1964) is compared with the performance of the albinos of the present investigation, strain differences which appear important remain. Adapted hooded Ss did not show the same atypical learning curve as the unadapted hooded Ss of this study; yet at every stage in learning they did make more errors than the albino of this investigation.

Although the present study provides no explanation for the discrepant results in this area, it does, along with others (Cross \& Boyer, 1964; Lawson et al., 1959), question the conclusion that, with the absolute method, quantitative variation in incentivew has no effect upon learning.

\section{Heferences}

CROSS, H. A., \& BOYER, W. N. Influence of amount of reward in a complex learning situation. Psychol. Rep., 1964, 14, 427-432.

LAWSON, R., CROSS, H. A., \& TAMBE, J. T. Effects of large and small rewards on maze performance after different prior experiences with reward amounts. J. comp. physiol. Psychol., 1959, 52, 717-720.

PUBOLS, B. H. Incentive magnitude, learning, and performance in animals. Psychol. Bull., 1960, 57, 89-115.

SCHRIER, A. M. Amount of incentive and performance in a black-white discrimination problem. J. comp. physiol. Psychol., 1956, 49, 123-125.

\section{Notes}

1. This research was supported in partby Grant No.154, from the Research Foundation of the Oklahoma State University.

2. Now at Texas Technological College.

3. The albinos were ordered from Sprague-Dawley (Madison, Wisc.); the hooded Ss from Rockland Farms (N. Y.). 